\title{
Grey Prediction on Rolling Bearing Friction Torque
}

\author{
Xintao $\mathrm{Xia}^{1} \& \mathrm{Tao} \mathrm{Ma}^{1}$ \\ ${ }^{1}$ College of Mechatronical Engineering, Henan University of Science and Technology, Luoyang, China \\ Correspondence: Xintao Xia, 63\#, Xiyuan Campus, Henan University of Science and Technology, 48 Xiyuan \\ Road, Luoyang 471003, China. Tel: 86-139-4929-7723. E-mail: xiaxt1957@163.com, xiaxt@mail.haust.edu.cn
}

Received: October 18, 2012 Accepted: November 7, 2012 Online Published: November 21, 2012

doi:10.5539/mer.v2n2p108

URL: http://dx.doi.org/10.5539/mer.v2n2p108

\begin{abstract}
The rolling bearing friction torque is forecasted by means of the grey dynamic model GM $(1,1)$. Residual test and posteriori error test are conducted to verify the reliability of the results of prediction. The experiment shows that the method proposed has the high precision and satisfy the engineering demand.
\end{abstract}

Keywords: grey dynamic model, rolling bearing, friction torque, prediction

\section{Introduction}

The friction torque as an important dynamic performance index is mainly used for evaluating the flexibility and the wear-life of rolling bearings in running.

So far, the research on the friction torque of rolling bearings has made great progress. Hernandez Montero and Medina (2007) considered four factors that cause rolling bearing friction, deduced theoretical calculation formulas of friction torque, and verified the reliability of formula based on the quasi static analysis. Townsend, Allen, and Zaratsky et al. (1973) studied the influence of different lubrication conditions on friction torque characteristics. Kitahara and Okamoto (1984) deduced an experience formula of the friction torque under the combined loading by the experimental investigation of precision bearings. Zhang and $\mathrm{Li}$ (2001) established the quasi kinetics analysis model and deduced the friction torque of ball bearings with the method of energy conversion. Gao and Xia (2012) reported that the friction torque of rolling bearings belongs to a poor information system, i.e., its probability distribution function and trend is unknown (Xia, Zhu, \& Lv, 2010). Wang (2008) studied the evaluation method for the friction torque of large size rolling bearings via the maximum entropy principle. Xia and Lv (2012) researched a dynamic prediction model for the rolling bearing friction torque using the grey bootstrap fusion method and the chaos theory.

At present the research about prediction of the rolling bearing friction torque is few due to poor information (Xia, Zhu, \& Lv, 2010). Poor information means incomplete information, such as, in system analysis, unknown trends and functions. Using the grey system theory, this work aims at prediction method for the friction torque of rolling bearings.

\section{Grey Prediction Model GM $(1,1)$}

Grey prediction (Deng, 1992), using existing data to build a grey system model, can infer the future development trend of the system. Its predicted value is a grey plane interval and the size of the interval is inversely proportional to the precision of prediction. The grey model GM $(1,1)$ conducts a preliminary treatment for the erratically limited data, using the processed data to establish the model, and then reveals the potential rule of the system.

Assume the original state sequence $X^{(0)}$ is given by

$$
X^{(0)}=\left(x^{(0)}(1), x^{(0)}(2), \ldots, x^{(0)}(i), \ldots, x^{(0)}(n)\right)
$$

where $x^{(0)}(i)$ is the $i$ th datum in $X^{(0)}$ and $n$ is the number of the data in $X^{(0)}$.

Defined the accumulating generation sequence as

$$
X^{(1)}=\left(x^{(1)}(1), x^{(1)}(2), \ldots, x^{(1)}(k), \ldots, x^{(1)}(n)\right)
$$


with

$$
x^{(1)}(k)=\sum_{i=1}^{k} x^{(0)}(i), k=1,2, \ldots, n
$$

The mean series of the close neighbours is defined as

$$
Z^{(1)}=\left(z^{(1)}(2), z^{(1)}(3), \ldots, z^{(1)}(n)\right)
$$

where

$$
z^{(1)}(k)=0.5 x^{(1)}(k)+0.5 x^{(1)}(k-1), k=2,3, \ldots, n
$$

So the grey differential equation can be given by

$$
x^{(0)}(k)+a z^{(1)}(k)=b, k=2,3, \ldots, n
$$

The whitening differential equation corresponding to equation (6) is

$$
\frac{\mathrm{d} x^{(1)}}{\mathrm{d} t}+a x^{(1)}(t)=b
$$

The solution to the whitening differential equation is

$$
\hat{x}^{(1)}(k+1)=\left(x^{(0)}(1)-\frac{b}{a}\right) e^{-a k}+\frac{b}{a}, k=1,2, \ldots, n-1
$$

where

$$
(a, b)^{\mathrm{T}}=\left(B^{\mathrm{T}} B\right)^{-1} B^{\mathrm{T}} Y
$$

with

$$
Y=\left(x^{(0)}(2), x^{(0)}(3), \ldots, x^{(0)}(n)\right)^{\mathrm{T}}
$$

and

$$
B=\left[\begin{array}{cc}
-z^{(1)}(2) & 1 \\
-z^{(1)}(3) & 1 \\
\vdots & \vdots \\
-z^{(1)}(n) & 1
\end{array}\right]
$$

According to the grey system theory, the reduction sequence is given by

$$
\hat{X}^{(0)}=\left(\hat{x}^{(0)}(1), \hat{x}^{(0)}(2), \ldots, \hat{x}^{(0)}(k), \ldots, \hat{x}^{(0)}(n)\right)
$$

where

$$
\hat{x}^{(0)}(k+1)=\hat{x}^{(1)}(k+1)-\hat{x}^{(1)}(k)
$$

\section{Test Model}

A grey model needs to be tested to determine whether it is reasonable. Only through the model test it can be used to predict. Two test methods are proposed in this work.

\subsection{Residual Test}

The residual sequence is defined as 


$$
\varepsilon=(\varepsilon(1), \varepsilon(2), \ldots, \varepsilon(n))=\left(x^{(0)}(1)-\hat{x}^{(0)}(1), x^{(0)}(2)-\hat{x}^{(0)}(2), \ldots, x^{(0)}(n)-\hat{x}^{(0)}(n)\right)
$$

The relative error sequence is defined as

$$
\Delta=(\Delta(1), \Delta(2), \ldots, \Delta(n))=\left(\left|\frac{\varepsilon(1)}{x^{(0)}(1)}\right|,\left|\frac{\varepsilon(2)}{x^{(0)}(2)}\right|, \ldots,\left|\frac{\varepsilon(n)}{x^{(0)}(n)}\right|\right)
$$

For $k \leq n$, the average relative error is given by

$$
\bar{\Delta}=\frac{1}{n} \sum_{k=1}^{n} \Delta(k)
$$

with

$$
\Delta(k)=\left|\frac{\varepsilon(k)}{x^{(0)}(k)}\right|
$$

The average precision is defined as

$$
p^{0}=(1-\bar{\Delta}) \times 100 \%
$$

\subsection{Posteriori Error Test}

Posteriori error test is a statistical concept, which is in accordance with the probability distribution of the residuals, to evaluate the accuracy of a model. Steps of posteriori error test are:

(1) Calculate the mean $\bar{x}$ and the variance $S_{1}^{2}$ of $X^{(0)}$ :

$$
\begin{gathered}
\bar{x}=\frac{1}{n} \sum_{k=1}^{n} x^{(0)}(k) \\
S_{1}^{2}=\frac{1}{n} \sum_{k=1}^{n}\left(x^{(0)}(k)-\bar{x}\right)^{2}
\end{gathered}
$$

(2) Calculate the mean $\bar{\varepsilon}$ and the variance $S_{2}^{2}$ of $\varepsilon$ :

$$
\begin{gathered}
\bar{\varepsilon}=\frac{1}{n} \sum_{k=1}^{n} \varepsilon(k) \\
S_{2}^{2}=\frac{1}{n} \sum_{k=1}^{n}\left(\varepsilon^{(0)}(k)-\bar{\varepsilon}\right)
\end{gathered}
$$

(3) Calculate the posterior error ratio $C$ and the small error probability $P$ :

$$
\begin{gathered}
C=\frac{S_{2}}{S_{1}} \\
P=\left\{|\varepsilon(k)-\bar{\varepsilon}| \leq 0.6745 S_{1}\right\}
\end{gathered}
$$

(4) Determine the precision of the model according to the precision grade shown in Table 1. 
Table 1. Precision scale

\begin{tabular}{ccc}
\hline Precision grade & $P$ & $C$ \\
\hline Good & $>0.95$ & $<0.35$ \\
Qualified & $>0.80$ & $<0.5$ \\
Barely qualified & $>0.70$ & $<0.65$ \\
Unqualified & $\leq 0.70$ & $\geq 0.65$ \\
\hline
\end{tabular}

\section{Case Study}

The experimental condition in this case study is listed in Table 2.

Table 2. Experimental condition

\begin{tabular}{ccc}
\hline Experiment rig and rolling bearing & Parameter & Parameter value \\
\hline Experiment rig & Axial load $/ \mathrm{N}$ & 20 \\
& Rotational speed of spindle/(r/min $)$ & 6 \\
Rolling bearing marked as HTKA & Bore diameter $/ \mathrm{mm}$ & 190 \\
& Outside diameter $/ \mathrm{mm}$ & 269.5 \\
& Width $/ \mathrm{mm}$ & 66 \\
& Weight $/ \mathrm{kg}$ & 12.8 \\
\hline
\end{tabular}

The experiment system consistes of the friction torque test subsystem, the axial loading subsystem, the speed regulating subsystem, and data acquisition and processing subsystem. Figure 1 is the structure schematic plan and the operating principle of the experiment rig of the rolling bearing friction torque.

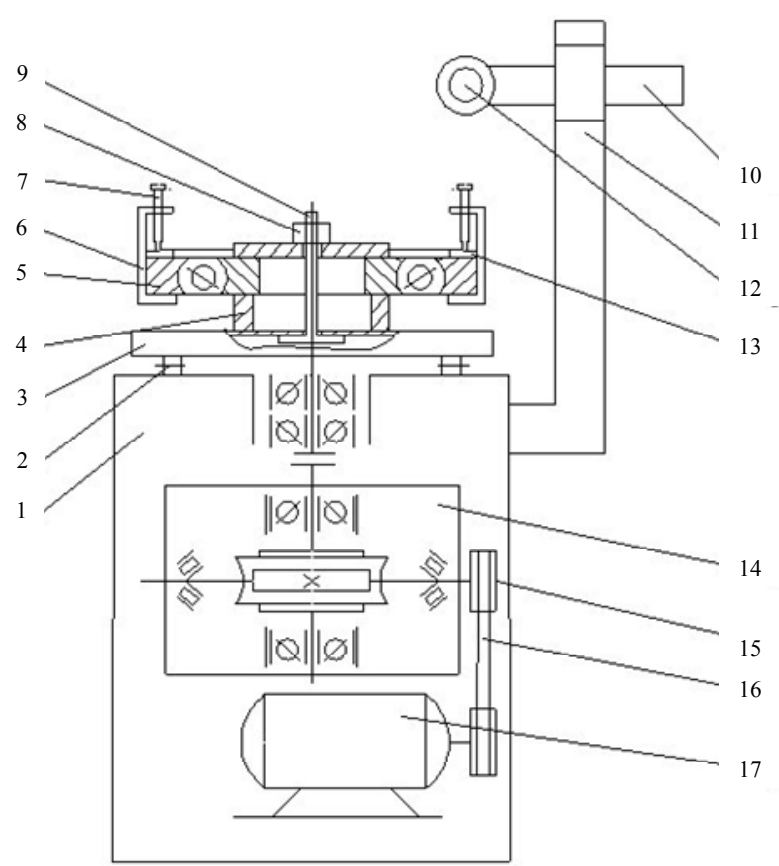

1 frame; 2 roller for rolling support; 3 turning table; 4 support seat; 5 tested rolling bearing; 6 fixture; 7 bolt; 8 clamp nut; 9 spindle; 10 force arm; 11 column; 12 pressure sensor;

13 weight; 14 gearbox; 15 pulley; 16 belt; 17 motor

Figure 1. Structure schematic plan and operating principle of friction torque test rig of rolling bearing 
As shown in Figure 1, during the measurement of the friction torque, the spindle drives the inner ring of the tested rolling bearing to rotate through the turning table, meanwhile, the outter ring that is subjected to the action of the friction torque turns in the same direction as the inner ring and then stops when the fixture mounted on the outter ring is blocked by the pressure sensor. The forces acting on the pressure sensor is in proportion to the friction torque, which can be expressed as the electrical signal, in $\mathrm{mA}$

Simulation test is did by using the experiment data of the rolling bearing friction torque for dynamic prediction under the condition of the modeling section $s=5$ (that is to say, the original data of sequence numbers 1 to 5 are used to predict the sixth datum, the original data of sequence numbers 2 to 6 are used to predict the seventh datum, ..., and, the original data of sequence numbers 96 to 100 are used to predict the 101 st datum). The results are shown in Figures 2 and 3.

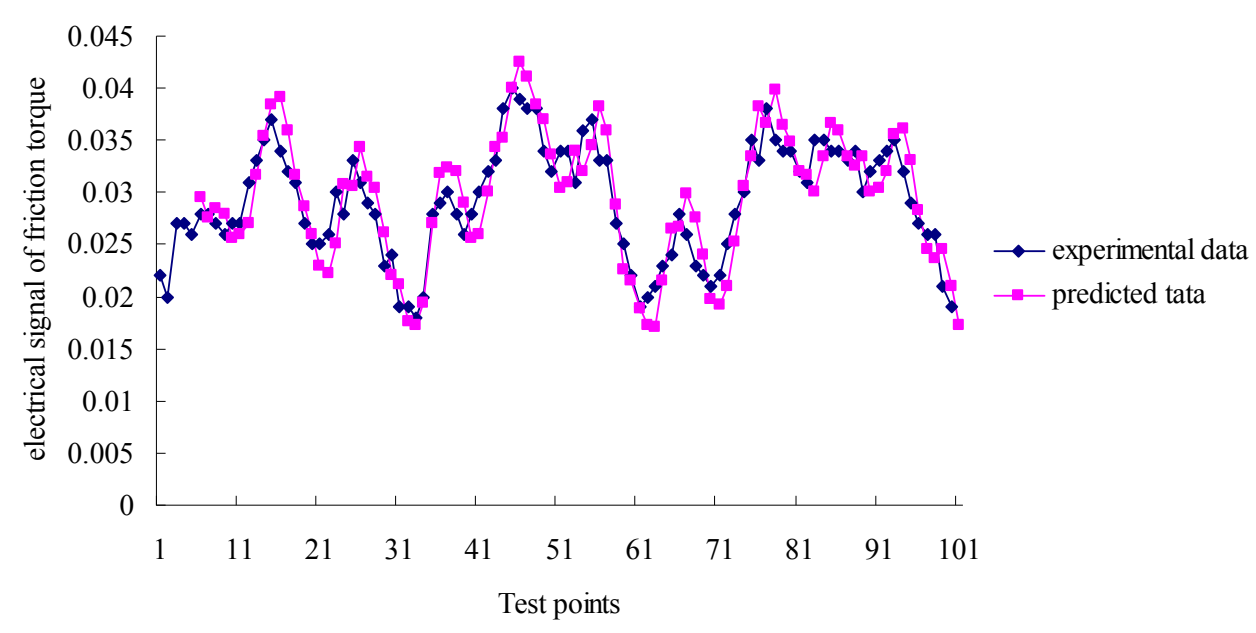

Figure 2. Experimental data and predicted data

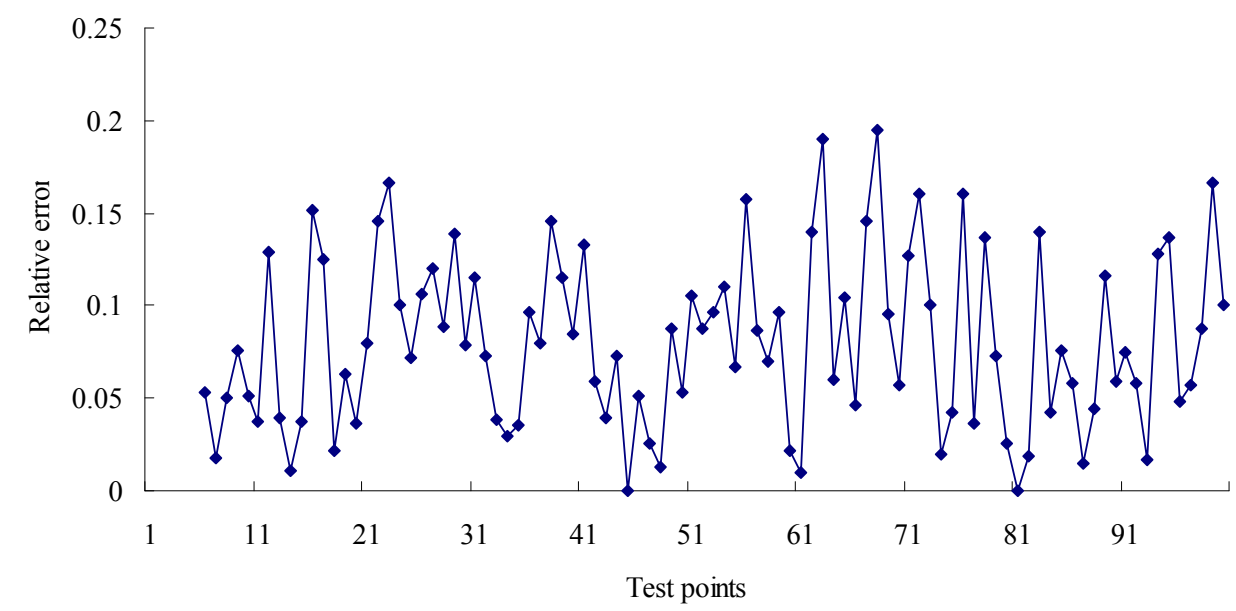

Figure 3. Relative error between experimental data and predicted data

It can be seen from Figures 2 and 3 that predicted values coincide with experimental values and the maximum and the mean of the relative errors between predicted values and experimental values is less than $20 \%$ and $10 \%$, respectively. In addition, the prediction precision can reach $90.8 \%$ and the effect is preferable. In the posteriori error test, the mean and variance of the original sequence are 0.029 and $28.7 \times 10^{-6}$, the mean and the variance of the residual error sequence are -0.0004 and $6.91 \times 10^{-6}$, so variance ratio $C$ is 0.488 , the precision grade is qualified, the small error probability $P$ is 0.85 , and the precision grade is also qualified. As a result, using the grey model on predicting the rolling bearing friction torque is reasonable and feasible, the accuracy is ideal, and 
the prediction data is reliable.

\section{Conclusion}

This work presents the grey prediction and precision test method for the rolling bearing friction torque. The experiment shows using the proposed method to predicte the rolling bearing friction torque is reasonable and feasible, the accuracy is ideal, and the prediction data is reliable.

\section{Acknowledgements}

This project was funded by the National Natural Science Foundation of China (Grant No. 51075123), the Natural Science Research Project of the Education Department of Henan Province (Grant No. 2010B460008), and the Doctoral Scientific Research Initiation Fund of Henan University of Science and Technology (Grant No. 09001318).

\section{References}

Deng, J. L. (1992). Grey system theory elements. Wuhan: Huazhong University of Science and Technology Press (in Chinese).

Hernandez Montero, F. E., \& Medina, O. C. (2008). The application of bispectrum on diagnosis of rolling element bearings: A theoretical approach. Mechanical Systems and Signal Processing, 22, 588-596. http://dx.doi.org/10.1016/j.ymssp.2007.09.003

Gao, L. L. \& Xia, X. T. (2012). Poor information analysis of rolling bearing friction torque characteristic parameter based on phase space. Advanced Materials Research, 382, 167-171. http://dx.doi.org/10.4028/www.scientific.net/AMR.382.167

Kitahara, T., \& Okamoto, J. (1984). Friction torque of instrument ball bearings under combined radial and thrust load. Japan Society of Lubrication Engineers, 5, 131-136.

Townsend, D. P., Allen, C. W., \& Zaratsky, E. V. (1973). Study of ball bearing torque under elastohydrodynamic lubrication. National Aeronautice and Space Administration, Lewis Research Center, Cleveland, Ohio, NASA-TM-X-68271.

Wang, C. X. (2008). Experimental Study on Friction Torque of Large Size Rolling Bearing. Luoyang: Henan University of Science and Technology (in Chinese).

Xia, X. T., \& Lv, T. M. (2012). Dynamic prediction model for rolling bearing friction torque using grey bootstrap fusion method and chaos theory. Advanced Materials Research, 443-444, 87-96. http://dx.doi.org/10.4028/www.scientific.net/AMR.443-444.87

Xia, X. T., Zhu, J. M., \& Lv, T. M. (2010). Poor information inference on friction torque of rolling bearings. Beijing: Science Press (in Chinese).

Zhang, K., \& Li, J. H. (2001). Analysis and calculation on friction torque of ball bearings. Bearing, 54(1), 8-11 (in Chinese). 